BMJ Open Diabetes Research \& Care

\title{
Quality of care in rural youth with type 1 diabetes: a cross-sectional pilot assessment
}

\author{
Kyle S Stumetz, ${ }^{1}$ Joyce P Yi-Frazier, ${ }^{2}$ Connor Mitrovich, ${ }^{3}$ Kathaleen Briggs Early ${ }^{4}$
}

To cite: Stumetz KS, YiFrazier JP, Mitrovich C, et al. Quality of care in rural youth with type 1 diabetes: a crosssectional pilot assessment BMJ Open Diabetes Research and Care 2016;4:e000300. doi:10.1136/bmjdrc-2016000300

Received 15 July 2016 Revised 3 October 2016 Accepted 6 November 2016

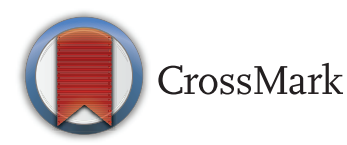

${ }^{1}$ PNWU, College of Osteopathic Medicine, Yakima, Washington, USA

${ }^{2}$ Seattle Children's Research Institute, Seattle, Washington, USA

${ }^{3}$ A.T. Still University of Health Sciences, Kirksville College of Osteopathic Medicine,

Kirksville, Missouri, USA

${ }^{4}$ Department of Biomedical Sciences, PNWU, College of Osteopathic Medicine, Yakima, Washington, USA

Correspondence to Dr Kathaleen Briggs Early; kearly@pnwu.edu

\section{ABSTRACT}

Background: Type 1 diabetes mellitus (T1DM) presents a significant health burden for patients and families. The quality of care (QOC) among those living in rural communities is thought to be subpar compared with those in urban communities; however, little data exist to reflect this, especially in pediatric diabetes.

Objective: The purpose of this pilot study was to investigate diabetes $Q O C$ among families living in rural versus urban areas. 6 QOC markers were used to compare youth with T1DM: appointment adherence, patient-provider communication, diabetes education during clinic visit, congruency with diabetes standards of care, diabetes self-management behaviors, and diabetes-related hospitalizations.

Research design and methods: Participants were rural or urban adult caregivers of youth ages 2-18 with $\geq 10$-month history of T1DM receiving treatment at Seattle Children's Hospital, USA. Participants were from rural areas of central Washington, or urban areas of western Washington. Caregivers completed a 26item survey pertaining to the $6 \mathrm{QOC}$ markers. The 6 QOC markers were compared across 61 participants (34 rural, 27 urban), to determine how diabetes care quality and experiences differed. Data were collected over 12 months. Groups were compared using t-tests and $\chi^{2}$ tests, as appropriate.

Results: Compared with urban families, rural families reported significantly lower income and a 4-fold greater usage of public insurance. Among the QOC measures, rural participants were significantly worse off in the appointment adherence, patient-provider communication, and hospitalizations categories. Congruence with diabetes standards of care (foot care only) was also significantly poorer in rural participants. Conclusions: The burden of travel in conjunction with the lack of resources in this rural population of families with T1DM youth is cause for concern and warrants further research.

\section{INTRODUCTION}

Type 1 diabetes mellitus (T1DM) in youth is a substantial health burden for the patient, their family, and the healthcare system. For patients living in rural or medically underserved communities, there is even greater

\author{
Significance of this study
}

What is already known about this subject?

- Disparities in quality of care among adults with diabetes are well recognized. Additionally, many understand that those living in rural communities have greater difficulty accessing optimal medical care. However, the impact on youth with type 1 diabetes, and their families who live several hours from their diabetes care team, is less well understood.

What are the new findings?

- Despite both groups receiving their diabetes care at a children's hospital, the rural youth in this study were found to have lower appointment adherence, less optimal communication with their care team, higher rates of diabetes-related hospitalizations, and were not getting care in agreement with American Diabetes Association (ADA) standards of care to the same degree as their urban counterparts.

How might these results change the focus of research or clinical practice?

- Rural youth with diabetes and their families encounter significant barriers to optimal diabetes care. Care teams should consider these obstacles and alternatives to meeting patient and family needs in designing care plans. Researchers should also consider the unique needs of rural youth when they recruit and design studies examining diabetes issues.

difficulty obtaining diabetes care that matches current clinical practice recommendations. ${ }^{1-3}$ Additionally, rural persons with diabetes are more likely to develop diabetesrelated complications. ${ }^{3-6}$ These inequities in quality of care (QOC) need addressing, particularly in the rural pediatric diabetes population where little about QOC is reported.

In the past 20 years, medical management of diabetes has greatly increased in complexity, and intensive diabetes treatment has been consistently shown to result in improved outcomes and reduced risk for complications. ${ }^{78}$ Intensive diabetes management, such as multiple daily injections or 
insulin pump therapy is associated with improved glycemic control and no greater risk of adverse effects among youth with T1DM. ${ }^{9} 10$ However, many rural physicians have limited access to the resources or technology to offer ongoing support to their patients. ${ }^{11}{ }^{12}$ Access to subspecialties such as pediatric endocrinology is also very limited in most rural communities, as is transportation for medical appointments. ${ }^{5}$ Additionally, diabetes hospitalizations have been shown to be more likely among lower socioeconomic youth with diabetes. ${ }^{4} 13$

QOC, defined by the Institutes of Medicine as healthcare that is 'safe, effective, patient-centered, timely, efficient and equitable', ${ }^{14}$ is determined by a number of factors including health status of the patient, physician experience, patient and parent preferences, geographic residence and socioeconomic status. ${ }^{12} 1516$ Given the economic $^{17}$ and psychosocial ${ }^{18}$ impact of T1DM in youth, it would be beneficial to understand how youth with T1DM and their families are affected by living in a rural or medically underserved community. Currently, little research is reported in this area. ${ }^{19}$

Thus, the primary objective of this cross-sectional pilot study was to assess the QOC reported by caregivers of youth with T1DM living in rural areas compared with those living in urban areas. The QOC markers used toward this aim were: (1) appointment adherence, (2) patient-provider communication, (3) diabetes education during clinic visit, (4) congruency with standards of care, (5) diabetes self-management behaviors, and (6) diabetes-related hospitalizations. Additionally, we compared diabetes-related clinical parameters (eg, glycemic control, medication/insulin regimen used, etc) among rural and urban patients. These findings are presented in accordance with the Strengthening the Reporting of Observational Studies in Epidemiology (STROBE) statement. ${ }^{20} 21$

\section{RESEARCH DESIGN AND METHODS \\ Participants}

A total of 61 participants were recruited and enrolled from families receiving care at Seattle Children's Hospital based on zip-code of residence by convenience sample. Although no universal definition exists, in accordance with the State of Washington's Office of Financial Management population density guidelines, families were further classified as either 'rural' or 'urban' based on whether their county had either more than or $<100$ people per square mile. ${ }^{22} 23$ This definition is commonly used in healthcare policy and research. ${ }^{24}{ }^{25}$ Participants were caregivers (eg, parent or guardian) of youth living with T1DM for at least 10 months beyond diagnosis and between the ages of 2 and 18. Eligible participants were contacted during regularly scheduled clinic appointments at the Seattle Children's Hospital in Seattle, Washington. Contact occurred by mail, phone, or in-person visit prior to their regular appointment. Data collection took 3 months for the urban group and 12 months for the rural group. The Institutional Review Board of Seattle Children's Hospital and Pacific Northwest University approved the protocol, and voluntary written informed consent was obtained from each caregiver. All participants completed a questionnaire packet, and received a $\$ 10$ gift card for their time. Medical record review was conducted for all clinical measures, including glycated hemoglobin (HbA1c), hospital admissions for diabetic ketoacidosis (DKA), and insulin regimen.

\section{QOC markers}

Perceptions of six markers for QOC were assessed using a 26-item survey adapted from the SEARCH For Diabetes in Youth Study's QOC survey, ${ }^{26}{ }^{27}$ which was similar to another large-scale diabetes QOC survey but is administered to countries (not patients or caregivers) on an annual basis. ${ }^{28}$ The six markers we used included: (1) appointment adherence, (2) patient-provider communication, (3) diabetes education during clinic visit, (4) congruency with standards of care, (5) diabetes selfmanagement behaviors, and (6) diabetes-related hospitalizations. Each caregiver completed the survey onsite during a clinical research center visit. The survey included a variety of questions reflecting each of the six overarching QOC markers, described in more detail below.

1. Appointment adherence: the number of appointments over the course of 12 months was recorded from the medical record. 'Adherence' was defined as four visits/year, which is the standard frequency recommended for routine follow-up. ${ }^{29}$

2. Patient-provider communication: four items were used to assess communication between the patient (or caregiver) and provider: 'How often did your provider: (1) offer you choices about your child's healthcare; (2) talk about the good and bad things of each choice for your child's healthcare; (3) ask you to tell them what choices you prefer; and (4) involve you as much as you wanted?' These were scored on a fourpoint Likert scale and a total patient-provider communication score was calculated by summing the scores of these four items (total range $=4-16$ ).

3. Diabetes education during clinic visit: This was assessed using seven items which align with the American Association of Diabetes Educators (AADE) 7 self-care behaviors, ${ }^{30}$ which are considered as quality indicators for diabetes care, ${ }^{31}$ structured as 'please indicate if a doctor or other health provider has talked to you/your child, about the following': (1) what to do for low blood sugar; (2) what to do for high blood sugar; (3) physical activity; (4) diet; (5) target blood sugar; (6) how to adjust insulin/ medication for sick days; and (7) psychological issues. The percentage of those affirming all seven areas of diabetes education during any of their clinic visits over the past 12 months was calculated. 
4. Congruency with standards of diabetes care: this included an assessment of care congruency with the American Diabetes Association Standards of Medical Care in use at the time of data collection. ${ }^{32}$ Areas evaluated included blood glucose, blood pressure, foot care, eye examination, kidney function, and blood lipids (eg, During the past 12 months, how often have your child's feet been checked during visits to your doctor's office?). The percentage of those affirming congruency with standards of diabetes care was calculated for each of the six areas.

5. Diabetes self-management behaviors: two specific aspects of diabetes self-management behaviors were assessed with the questions, 'In the past 3 months, how often has your child tested his/her blood sugar?' and 'In the past 3 months, how often does your child miss insulin or medication doses?' The percentage of those affirming that blood sugar testing was occurring at least four times per day, and that no insulin or medication doses were missed, was reported.

6. Diabetes-related hospitalizations: using data from the medical record, we identified those participants with reported incidences of DKA or diabetes-related hospitalizations over the past 12 months. The percentage was calculated for the total sample, and for rural and urban participants.

Finally, rural participants were asked to assess the level of hardship (barriers) on families to get patients to Seattle for care (eg, Coming to Seattle to get diabetes care for my child is a hardship for me/my family) scored on a scale of 0 (none of the time) to 4 (all of the time). For those responding affirmatively, they were asked in open-ended format to describe why they continue to seek care in Seattle, despite the hardships. For those who traveled from rural areas, additional follow-up questions were asked about how much of a hardship it was for families to travel to Seattle for care. Respondents from the Seattle area were not asked this question because they all lived within close proximity to the clinic. Open-ended, qualitative feedback about the type of hardship, and why travel occurred was also assessed for those affirming that travel was a hardship some of the time, most of the time, or all of the time.

Clinical information, including insulin regimen, HbA1c level, and diabetes-related hospitalizations, was gathered via electronic medical record review.

\section{Statistical analyses}

Descriptive data including means, SDs, ranges, and percentages were used to describe the overall study population. Student's t-tests and $\chi^{2}$ tests were used to compare the urban and rural groups on the key variables including demographic, clinical, and QOC markers. Significance was set to $\mathrm{p}<0.05$ and SPSS V.19 was used for all analyses.

\section{RESULTS}

\section{Patient population}

Demographic and clinical characteristics are shown in table 1. Rural and urban groups did not differ significantly in terms of age of the youth with diabetes, ethnicity/race, caregiver education level, use of an insulin pump, or family size (table 1). However, in contrast to urban families of youth with T1DM, rural families in the study reported significantly lower income $(p=0.05)$, and were almost four times as likely to have public insurance ( $\mathrm{p}=0.001)$.

\section{Differences in QOC between urban and rural participants}

Table 2 shows the six QOC markers for the overall sample stratified by rural or urban group. Appointment adherence, defined as making clinic visits as scheduled, was statistically different across the two groups with fewer rural participants meeting expected appointments ( $p=0.01$ ). Patient-provider communication scores were rated lower in rural compared with urban participants $(p<0.05)$. Finally, compared with those living in urban areas, there were more frequent diabetes-related hospitalizations and a greater perceived burden for families living in rural areas to obtain care in Seattle $(\mathrm{p}=0.005)$.

\section{Table 1 Demographic and clinical characteristics}

\begin{tabular}{|c|c|c|c|}
\hline & Total sample $(n=61)$ & Rural $56 \%(n=34)$ & Urban $44 \%(n=27)$ \\
\hline \multicolumn{4}{|l|}{ Demographic characteristics } \\
\hline Age of participating youth (mean $\pm S D$ ) & $13.3 \pm 3.4$ & $13.2 \pm 3.4$ & $13.5 \pm 3.6$ \\
\hline Race $(\% \mathrm{NHW})$ & $82 \%$ & $74 \%$ & $93 \%$ \\
\hline Education level of parent/caregiver (>high school) & $68 \%$ & $63 \%$ & $74 \%$ \\
\hline Family income $(\%<\$ 50 \mathrm{~K} /$ year $)$ & $36 \%$ & $47 \%$ & $22 \%$ * \\
\hline Public insurance & $38 \%$ & $56 \%$ & $15 \%$ ** \\
\hline \multicolumn{4}{|l|}{ Clinical characteristics } \\
\hline HbA1c (mean \pm SD) & $8.8 \pm 1.7$ & $9.0 \pm 1.8$ & $8.5 \pm 1.6$ \\
\hline Insulin regimen (\%pump) & $54 \%$ & $47 \%$ & $63 \%$ \\
\hline Duration of diabetes (mean $\pm S D)$ & $5.4 \pm 3.2$ & $4.9 \pm 3.2$ & $6.1 \pm 3.0$ \\
\hline
\end{tabular}




\begin{tabular}{|c|c|c|c|}
\hline & $\begin{array}{l}\text { Percentages or mean } \\
\text { (SD) }(n=61)\end{array}$ & $\begin{array}{l}\text { Rural } \\
(n=34)\end{array}$ & $\begin{array}{l}\text { Urban } \\
(n=27)\end{array}$ \\
\hline 1. Appointment adherence & $72 \%$ & $59 \%$ & $89 \% *$ \\
\hline 2. Patient-provider communication (SD) & $13.6(2.7)$ & $13.0(3.0)$ & $14.4(2.2)^{*}$ \\
\hline 3. Diabetes education during clinic visit & $65 \%$ & $61 \%$ & $70 \%$ \\
\hline \multicolumn{4}{|l|}{ 4. Congruency with 2014 ADA standards of care } \\
\hline A. Blood glucose monitoring & $100 \%$ & $100 \%$ & $100 \%$ \\
\hline B. Blood pressure & $87 \%$ & $65 \%$ & $93 \%$ \\
\hline C. Foot care & $74 \%$ & $65 \%$ & $89 \% *$ \\
\hline D. Eye examination & $50 \%$ & $44 \%$ & $59 \%$ \\
\hline E. Kidney function & $31 \%$ & $24 \%$ & $41 \%$ \\
\hline F. Blood lipids & $52 \%$ & $50 \%$ & $56 \%$ \\
\hline $\begin{array}{l}\text { 5. Diabetes self-management behaviors (\% not missing prescribed } \\
\text { insulin/medication dosing, or SMBG) }\end{array}$ & $60 \%$ & $71 \%$ & $52 \%$ \\
\hline 6. Diabetes-related hospitalization or DKA incidence in the past year & $19 \%$ & $33 \%$ & $4 \%$ ** \\
\hline
\end{tabular}

Diabetes education during clinic visit was without significant difference between groups. Rates of access to various healthcare disciplines (ie, registered dietitian, social work, etc) was the same for rural and urban participants.

Congruency with diabetes standards of care was not significantly different except for foot care, where $89 \%$ of urban and $65 \%$ of rural respondents endorsed that feet were checked at least once during the past 12 months $(\mathrm{p}=0.03)$. All other expected areas related to the standards of diabetes care and diabetes education were perceived as adequate regardless of rural or urban participant.

\section{Exploring the burden of travel among rural participants}

When rural participants were asked about their reasons for traveling so far (some exceeding 4 hours), $44 \%$ endorsed, 'it is worth it to receive the best possible care' and 33\% chose, 'no other option'. When asked to describe in more detail the hardships experienced due to travelling so far for care, qualitative responses included:

Requires 3-hour trip each way and sometimes an overnight stay. My son has to miss a full day of high school [and] sports practice. We have had extra trips to start pump use.

I do not have the time available for my work.

Having to miss work, cost of fuel and find someone to watch dogs during trip.

Because it is far away and I have to ask someone else to drive ... and I have to leave my other kids.

Driving, wear and tear on car, winter weather conditions, missing a day of school/work.

The majority $(67 \%)$ of the rural participants endorsed that travel was a hardship, particularly in regards to the financial impact of travel and time-off from work, and some reported concerns over the QOC available locally:
Only care/best care [available].

No provider out in [my town], they don't know as much as they do at Seattle Children's.

Very good care. None available in [my town]. We want [our] son to learn good management.

Because I do not know if there is a specialist in my community.

No quality care in [my town].

Because in [my town] there are no specialists for kids.

\section{DISCUSSION}

Our analysis explored six markers of QOC among patients and families from rural versus urban communities. Appointment adherence, patient-provider communication, diabetes-related hospitalizations and one of the measures of congruency with diabetes standards of care (foot care) were all found to be significantly poorer among the rural respondents in our survey.

Appointment adherence has been linked to poor diabetes care outcomes in linear fashion such that as appointment adherence worsens, HbA1c increases along with a greater overall risk of hospitalization and developing DKA. ${ }^{33} 34$

Similarly, patient-provider communication has long been recognized as a factor in patient satisfaction and adherence to treatment recommendations. ${ }^{35-38}$ Good communication between the healthcare team and the patient-family unit is key to motivating patients and help them feel providers have recognized their achievements and struggles. ${ }^{35}{ }^{39}$ Our findings emphasize the elevated risk for poor outcomes that can be augmented in high-risk communities, such as those with chronic disease living in rural locations. The distance to care is not only impacting attendance at regular visits, but 
perceived communication between the healthcare team and the patient and their family, placing these patients at elevated risk for poor outcomes. This is consistent with health disparities research showing poorer outcomes for rural patients with diabetes or other chronic diseases. ${ }^{3-6}$ Overcoming communication barriers is critical and using technologies such as phone, email or video/online conferencing could provide opportunities to reduce this disparity. ${ }^{40} 41$

The finding that foot care did not meet the diabetes standards of care was surprising, especially given that all other areas met the standards. Peripheral neuropathy is a serious concern among all persons with diabetes, but screening for it early in the disease course is essential, even for youth. ${ }^{42}$ Adult studies have found those with diabetes from rural areas have higher rates of peripheral neuropathy, ${ }^{43}$ but it is unclear if this is also a contributing factor in youth with diabetes.

The disparity in diabetes-related hospitalizations and DKA among our sample is especially concerning, but unfortunately not surprising. Hospitalizations related to diabetes increase with age and disease duration, and children and young adults account for about $40 \%$ of hospitalizations, ${ }^{44}{ }^{45}$ so finding effective ways to reduce these is imperative. DKA is well-recognized as a major and frequent complication for youth with established diabetes. ${ }^{46}$ Factors that contribute to more frequent hospital admissions might include less familiarity with diabetes and limited access to endocrinology expertise in a rural area, making it more difficult to determine when a situation requires hospital admittance. Other possible contributors could be difficulty understanding how to trouble-shoot blood sugars outside the target range; healthcare system issues such as potentially more reliance in emergency services rather than preventative care; financial barriers to diabetes supplies or optimal therapies; and psychosocial issues such as family dynamics. Our findings align with other studies that have reported increased medical resource usage and treatment burden among those from lower socioeconomic groups and rural persons with chronic disease. ${ }^{44} 4849$ Partnering with rural health clinics to optimize care at the local level may be one way to also positively affect hospitalization rates in this population. ${ }^{2}{ }^{6}$

Limitations of this pilot study include that it was a small, regional convenience sample, which is inherently susceptible to bias and not broadly generalizable. Further, some of the survey questions lacked time-frame specificity. Some of our QOC markers were derived from a study tool that despite being used in one of the largest studies of youth with diabetes, ${ }^{26} 27$ is not standardized or validated. Given that all participants received their care at a children's hospital, we may have found different results with a rural sample receiving care locally. The statistical power is low as demonstrated by the results reported in tables 1 and 2, which makes the identification of confounders/co-variates very difficult. Additionally, we are unaware of a patient-level validated diabetes QOC survey for youth and/or their patients, which would have enhanced the study design.

\section{CONCLUSIONS}

This pilot study sheds light on important differences between families living in rural versus urban areas, who have a child with T1DM. Getting to a provider several hours' drive away is a substantial burden for these families. Especially of note are the clinical implications, including more frequent episodes of DKA episodes and hospital admissions, among this sample of rural youth with T1DM.

${ }^{26}$ To reduce the disparities between these groups, regional and local healthcare groups need to provide support and offer practical solutions for families with diabetes living in rural areas. Including telehealth or web-based medical and educational services ${ }^{50}$ which are not well developed in this region, could likely offer a feasible alternative to long drives for medical appointments. Offering creative educational approaches that include a strong psychosocial support aspect could be a cost-effective and patient-centered approach. ${ }^{51}$

This pilot-level observational study illustrates the feasibility of assessing QOC where little data have been collected-among rural American youth with T1DM. Future studies should consider the unique needs of rural patients with diabetes in intervention efforts focused on serving, educating or delivering tailored diabetes care to reduce disparities between urban and rural residents.

Acknowledgements The authors wish to thank the participants in our study, the clinical research team at Seattle Children's, and the PNWU Seed Fund, which sponsored the project. A preliminary version of this work was presented in abstract and poster form at the 75th ADA Scientific Sessions in Boston, Massachusetts, USA, June 2015.

Contributors All authors contributed to the interpretation of the findings, drafting, revising of the manuscript, and approving the final draft. CM did the bulk of recruiting and collected most of the data. JPY-F and CM analyzed the data. JPY-F and KBE conceived the study design and wrote the initial proposal.

Funding PNWU Seed Fund.

Competing interests None declared.

Ethics approval PNWU IRB and Seattle Children's IRB.

Provenance and peer review Not commissioned; externally peer reviewed.

Data sharing statement No additional data are available.

Open Access This is an Open Access article distributed in accordance with the Creative Commons Attribution Non Commercial (CC BY-NC 4.0) license, which permits others to distribute, remix, adapt, build upon this work noncommercially, and license their derivative works on different terms, provided the original work is properly cited and the use is non-commercial. See: http:// creativecommons.org/licenses/by-nc/4.0/

\section{REFERENCES}

1. Coon P, Zulkowski K. Adherence to American Diabetes Association standards of care by rural health care providers. Diabetes Care 2002;25:2224-9.

2. Kirkbride K, Wallace N. Rural health clinics and diabetes-related primary care for Medicaid beneficiaries in Oregon. $J$ Rural Health 2009;25:247-52.

3. Krishna S, Gillespie KN, McBride TM. Diabetes burden and access to preventive care in the rural United States. J Rural Health 2010;26:3-11. 
4. Rewers A, Chase HP, Mackenzie T, et al. Predictors of acute complications in children with type 1 diabetes. JAMA 2002;287:2511-18.

5. Gamm L, Hutchison L, Dabney B, Dorsey A, eds. Rural healthy people 2010: a companion document to healthy people 2010. Vol 1. College Station, TX: The Texas A\&M University System Health Science Center, School of Rural Public Health, Southwest Rural Health Research Center, 2003. https://sph.tamhsc.edu/srhrc/docs/ rhp-2010-volume1.pdf

6. Siminerio LM, Piatt G, Zgibor JC. Implementing the chronic care model for improvements in diabetes care and education in a rural primary care practice. Diabetes Educ 2005;31:225-34.

7. Diabetes Control Complications Trial (DCCT)/Epidemiology of Diabetes Interventions Complications (EDIC) Study Research Group. Intensive diabetes treatment and cardiovascular outcomes in type 1 diabetes: The DCCT/EDIC study 30-year follow-up. Diabetes Care 2016;39:686-93.

8. Garvey KC, Telo GH, Needleman JS, et al. Health care transition in young adults with type 1 diabetes: perspectives of adult endocrinologists in the U.S. Diabetes Care 2016;39:190-7.

9. Orr CJ, Hopman W, Yen JL, et al. Long-term efficacy of insulin pump therapy on glycemic control in adults with type 1 diabetes mellitus. Diabetes Technol Ther 2015;17:49-54.

10. Phillip $\mathrm{M}$, Battelino $\mathrm{T}$, Rodriguez $\mathrm{H}$, et al. Use of insulin pump therapy in the pediatric age-group: consensus statement from the European Society for Paediatric Endocrinology, the Lawson Wilkins Pediatric Endocrine Society, and the International Society for Pediatric and Adolescent Diabetes, endorsed by the American Diabetes Association and the European Association for the Study of Diabetes. Diabetes Care 2007;30:1653-62.

11. Johnson JA, Eurich DT, Toth EL, et al. Generalizability and persistence of a multifaceted intervention for improving quality of care for rural patients with type 2 diabetes. Diabetes Care 2005;28:783-8.

12. Brundisini F, Giacomini M, DeJean D, et al. Chronic disease patients' experiences with accessing health care in rural and remote areas: a systematic review and qualitative meta-synthesis. Ont Health Technol Assess Ser 2013;13:1-33.

13. Shulman R, Stukel TA, Miller FA, et al. Low socioeconomic status is associated with adverse events in children and teens on insulin pumps under a universal access program: a population-based cohort study. BMJ Open Diabetes Res Care 2016;4:e000239.

14. Committee on Quality of Health Care in America, Institute of Medicine. Crossing the quality chasm: a new health system for the 21st century, 2001. https://www.nap.edu/read/10027/chapter/1

15. Frindik JP, Williams JP, Johnson RL, et al. Parental perception and factors associated with glycemic control in pediatric patients with type 1 diabetes. Endocr Pract 2002;8:105-8.

16. Redondo MJ, Connor CG, Ruedy KJ, et al., Pediatric Diabetes Consortium. Pediatric diabetes consortium type 1 diabetes New Onset (NeOn) study: factors associated with $\mathrm{HbA1c}$ levels one year after diagnosis. Pediatr Diabetes 2014;15:294-302.

17. Altamirano-Bustamante N, Islas-Ortega L, Robles-Valdés C, et al. Economic family burden of metabolic control in children and adolescents with type 1 diabetes mellitus. J Pediatr Endocrinol Metab 2008;21:1163-8.

18. Hood KK, Beavers DP, Yi-Frazier J, et al. Psychosocial burden and glycemic control during the first six years of diabetes: results from the SEARCH for diabetes in youth study. J Adolesc Health 2014;55:498-504.

19. Christakis DA, Feudtner C, Pihoker C, et al. Continuity and quality of care for children with diabetes who are covered by Medicaid. Ambul Pediatr 2001;1:99-103.

20. von Elm E, Altman DG, Egger M, et al. The Strengthening the Reporting of Observational Studies in Epidemiology (STROBE) statement: guidelines for reporting observational studies. Int J Surg 2014; 12:1495-9.

21. Bennett C, Khangura S, Brehaut JC, et al. Reporting guidelines for survey research: an analysis of published guidance and reporting practices. PLoS Med 2011;8:e1001069.

22. Washington State Department of Health. Primary care health professional shortage area by county. Secondary Primary Care Health Professional Shortage Area by County, 2016. http://www.doh. wa.gov/Portals/1/Documents/2900/689141.pdf

23. Washington State Office of Financial Management. Population density and land area criteria used for rural area assistance and other programs. Secondary Population density and land area criteria used for rural area assistance and other programs, 2012. http://www. ofm.wa.gov/pop/popden/rural.asp

24. Hart LG, Larson EH, Lishner DM. Rural definitions for health policy and research. Am J Public Health 2005;95:1149-55.
25. US Department of Health and Human Services (DHHS). Defining rural population. Secondary Defining Rural Population, 2015. http:// www.hrsa.gov/ruralhealth/aboutus/definition.html

26. Waitzfelder B, Pihoker C, Klingensmith G, et al. Adherence to guidelines for youths with diabetes mellitus. Pediatrics 2011;128:531-8.

27. Jaacks LM, Bell RA, Dabelea $D$, et al, SEARCH for Diabetes in Youth Study Group. Diabetes self-management education patterns in a US population-based cohort of youth with type 1 diabetes. Diabetes Educ 2014;40:29-39.

28. Schwarz PE, Gallein G, Ebermann D, et al. Global Diabetes Survey -an annual report on quality of diabetes care. Diabetes Res Clin Pract 2013;100:11-18.

29. Chiang JL, Kirkman MS, Laffel LM, et al. Type 1 diabetes through the life span: a position statement of the American Diabetes Association. Diabetes Care 2014;37:2034-54

30. AADE. AADE7 self-care behaviors. Diabetes Educ 2008;34:445-9

31. Wylie-Rosett J, Tobin JN, Davis N. Revised 2005 diabetes quality improvement checklist. Diabetes Educ 2005;31:669-78.

32. American Diabetes Association. Standards of medical care in diabetes-2014. Diabetes Care 2014;37(Suppl 1):S14-80.

33. Schectman JM, Schorling JB, Voss JD. Appointment adherence and disparities in outcomes among patients with diabetes. J Gen Intern Med 2008;23:1685-7

34. Fortin K, Pries E, Kwon S. Missed medical appointments and disease control in children with type 1 diabetes. J Pediatr Health Care 2016;30:381-9.

35. Young SA, Azam LS, Meurer JR, et al. The influence of patient and provider communication on diabetes care delivery. J Ambul Care Manage 2016;39:272-8.

36. Powell PW, Corathers SD, Raymond J, et al. New approaches to providing individualized diabetes care in the 21st century. Curr Diabetes Rev 2015;11:222-30.

37. Monaghan M, Hilliard M, Sweenie R, et al. Transition readiness in adolescents and emerging adults with diabetes: the role of patient-provider communication. Curr Diab Rep 2013;13:900-8.

38. Delamater AM. Improving patient adherence. Clin Diabetes 2006;24:71-7

39. Ayala J, Howe C, Dumser S, et al. Partnerships with providers: reflections from parents of children with type 1 diabetes. West J Nurs Res 2014;36:1238-53.

40. Franc S, Daoudi A, Mounier S, et al. Telemedicine and diabetes: achievements and prospects. Diabetes Metab 2011;37:463-76.

41. Gentles SJ, Lokker C, McKibbon KA. Health information technology to facilitate communication involving health care providers, caregivers, and pediatric patients: a scoping review. J Med Internet Res 2010;12:e22.

42. Jaiswal M, Lauer A, Martin CL, et al. Peripheral neuropathy in adolescents and young adults with type 1 and type 2 diabetes from the SEARCH for diabetes in youth follow-up cohort: a pilot study. Diabetes Care 2013;36:3903-8.

43. Wang W, Balamurugan A, Biddle J, et al. Diabetic neuropathy status and the concerns in underserved rural communities: challenges and opportunities for diabetes educators. Diabetes Educ 2011;37:536-48.

44. Kim S. Burden of hospitalizations primarily due to uncontrolled diabetes: implications of inadequate primary health care in the United States. Diabetes Care 2007;30:1281-2.

45. Lee JM, Okumura MJ, Freed GL, et al. Trends in hospitalizations for diabetes among children and young adults: United States, 1993 2004. Diabetes Care 2007;30:3035-9.

46. Bismuth $\mathrm{E}$, Laffel L. Can we prevent diabetic ketoacidosis in children? Pediatr Diabetes 2007;8(Suppl 6):24-33.

47. Smith $C P$, Firth $D$, Bennett $S$, et al. Ketoacidosis occurring in newly diagnosed and established diabetic children. Acta Paediatr 1998;87:537-41.

48. Kobos E, Imiela J. Factors affecting the level of burden of caregivers of children with type 1 diabetes. Appl Nurs Res 2015;28:142-9.

49. Galler A, Lindau M, Ernert A, et al. Associations between media consumption habits, physical activity, socioeconomic status, and glycemic control in children, adolescents, and young adults with type 1 diabetes. Diabetes Care 2011;34:2356-9.

50. Balkhi AM, Reid AM, Westen SC, et al. Telehealth interventions to reduce management complications in type 1 diabetes: a review. World J Diabetes 2015;6:371-9.

51. Sawtell M, Jamieson L, Wiggins M, et al. Implementing a structured education program for children with diabetes: lessons learnt from an integrated process evaluation. BMJ Open Diabetes Res Care 2015;3:e000065. 